\title{
Even-Denominator Filling Factors in the Thermoelectric Power of a Two-Dimensional Electron Gas
}

\author{
B. Tieke, ${ }^{1}$ U. Zeitler, ${ }^{1}$ R. Fletcher, ${ }^{2}$ S. A. J. Wiegers, ${ }^{1}$ A. K. Geim, ${ }^{1}$ J.C. Maan, ${ }^{1}$ and M. Henini ${ }^{3}$ \\ ${ }^{1}$ Research Institute for Materials, High Field Magnet Laboratory, University of Nijmegen, 6500 GL Nijmegen, The Netherlands \\ ${ }^{2}$ Physics Department, Queen's University, Kingston, Ontario, Canada K7L 3N6 \\ ${ }^{3}$ Department of Physics, University of Nottingham, Nottingham NG7 2RD, United Kingdom
}

(Received 22 December 1995)

\begin{abstract}
We have investigated the interaction of phonons with a two-dimensional electron gas in the fractional quantum Hall regime with phonon drag thermoelectric power (TEP). The TEPs at filling factors with the same even denominator are found to be identical; at other even-denominator filling factors they differ only by a constant factor. Treating these states as composite fermions, the findings are explained by a zero magnetic field theory for phonon drag. A maximum observed in the TEP at $\nu=\frac{1}{2}$ is modeled by a weakly diverging effective mass of the composite fermions at this filling factor. [S0031-9007(96)00178-0]
\end{abstract}

PACS numbers: 73.40.Hm, 72.20.Pa

New ground states appear in the energy spectrum of a two-dimensional electron gas (2DEG) at high magnetic fields and low temperatures when an odd fraction of Landau levels is occupied, an effect known as the fractional quantum Hall effect (FQHE). In a recently developed model $[1,2]$, the FQHE is attributed to quantum oscillations of new quasiparticles, called composite fermions (CFs), consisting of electrons to which an even number $2 m$ of flux quanta is attached. A profound consequence is that, at exactly even-denominator filling factors $\nu=\frac{1}{2 m}$, these new quasiparticles behave as fermions in a magnetic field which is effectively zero. This interpretation has proved to be highly successful in describing experimental results, including transport $[3,4]$, geometrical resonances in samples with antidots [5], and in surface acoustic wave experiments [6].

In this Letter we report thermoelectric experiments which probe the interaction of these quasiparticles with phonons. For a broad range of temperatures and electron densities, the experimental results convincingly demonstrate that states observed at half-filled Landau levels have the same value of the thermoelectric power (TEP), and that states at quarter filling are also related in a similar way. These results find an elegant explanation in terms of a zero-field Boltzmann theory for the phonon drag TEP with the appropriate CF parameters.

TEP can be used to study the interaction of phonons with 2DEGs at temperatures below $4 \mathrm{~K}$, where the dominant mechanism is phonon drag and also where the FQHE can be observed. Therefore, this technique provides information on the CF-phonon interaction [7]. At the very lowest temperatures, the phonons are frozen out and diffusion TEP, which has been observed in 2D hole gases [8], dominates, but this is not relevant in the present case. Phonon drag TEP is due to the flow of phonons in an applied temperature gradient which produces an electron current caused by electron-phonon interaction. Since no net current is drawn from the sample during measure- ments, an electric field appears which provides a compensating electric current, and the ratio of this electric field to the temperature gradient is the TEP. It is important to note that both the drag and compensation current are proportional to the same impurity relaxation time $\tau_{i}$, so that TEP becomes independent of this quantity. Thus phonon drag TEP is fundamentally different from the resistivity $\rho_{x x}$ which is almost completely determined by $\tau_{i}$ at low temperatures.

The TEP of a high mobility GaAs-GaAlAs heterostructure was measured at temperatures down to $150 \mathrm{mK}$ in magnetic fields up to $20 \mathrm{~T}$. The density and mobility of the 2DEG were varied in the range $n_{e}=(1.0-1.9) \times$ $10^{15} \mathrm{~m}^{-2}$ and $\mu=60-100 \mathrm{~m}^{2} / \mathrm{V} \mathrm{s}$, respectively, using illumination from an infrared diode. For TEP measurements, a calibrated temperature gradient was applied to the sample and the thermally created electric field measured. For this purpose a heater and two thermometers were glued to the substrate which was mounted on a cold finger in the vacuum space of a dilution refrigerator. The known thermal conductivity of the substrate was used as a check of the thermometry in both zero and high magnetic fields [7]. We will concentrate on $S_{x x}$, the component of the TEP along the direction of the temperature gradient.

Figure 1 shows the experimental magnetic field dependence of $S_{x x}$ at two different temperatures. As commonly observed for $\rho_{x x}$, we also see that for $S_{x x}$ the minima in the $\mathrm{FQH}$ regime are deepened as the temperature is lowered. However, Fig. 1 also shows the extraordinary result that the relative magnitude of the TEPs at evendenominator filling factors $\nu=\frac{1}{2}, \frac{3}{2}$, and $\frac{3}{4}$ is independent of temperature, with the first pair actually being identical within experimental error.

This striking experimental result can be seen more clearly in Fig. 2, where the ratios of $S_{x x}\left(\frac{3}{2}\right)$ and $S_{x x}\left(\frac{3}{4}\right)$ to $S_{x x}\left(\frac{1}{2}\right)$ are shown as a function of the inverse temperature. Both ratios increase as temperature decreases, but below a characteristic temperature $T_{c}$ they 


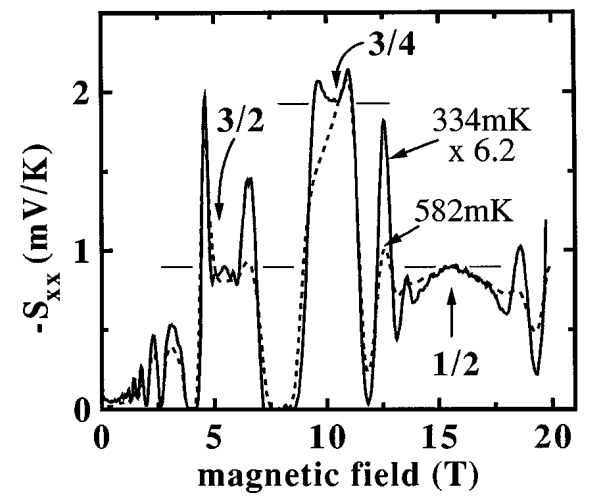

FIG. 1. Examples of the magnetic field dependence of the longitudinal component $S_{x x}$ of the TEP. The horizontal lines mark the constant relative value for the even-denominator filling factors $\nu=\frac{3}{2}$ and $\frac{3}{4}$.

remain constant corresponding to $S_{x x}\left(\frac{3}{2}\right) / S_{x x}\left(\frac{1}{2}\right)=$ $0.99 \pm 0.03$ and $S_{x x}\left(\frac{3}{4}\right) / S_{x x}\left(\frac{1}{2}\right)=2.15 \pm 0.05$. At an electron density $n_{e}=1.76 \times 10^{15} \mathrm{~m}^{-2}, T_{c}=480 \mathrm{mK}$ for both $\nu=\frac{3}{2}$ and $\frac{3}{4}$, which coincides with the temperature at which features of the FQHE around $\nu=\frac{3}{2}$ become visible (e.g., minima at $\nu=\frac{5}{3}$ and $\frac{4}{3}$ ).

We note that the constant relative TEP below $T_{c}$ is unique to the even-denominator filling factors $\nu=\frac{3}{2}$ and $\frac{3}{4}$. The TEPs at other filling factors in the FQH regime clearly have different temperature dependences; an example is given in Fig. 2. A similar correspondence between even-denominator filling factors is not observed in $\rho_{x x}$, e.g., in our sample we find $\rho_{x x}\left(\frac{3}{2}\right) / \rho_{x x}\left(\frac{1}{2}\right) \approx 0.2$.

This behavior of the TEP is found for all electron densities $n_{e}$ studied, the only variation being that $T_{c}$ increases with density. At lower electron densities the $\nu=\frac{1}{4}$ state becomes visible below $20 \mathrm{~T} . S_{x x}$ shows an almost field-independent plateau around $\nu=\frac{1}{4}$ as

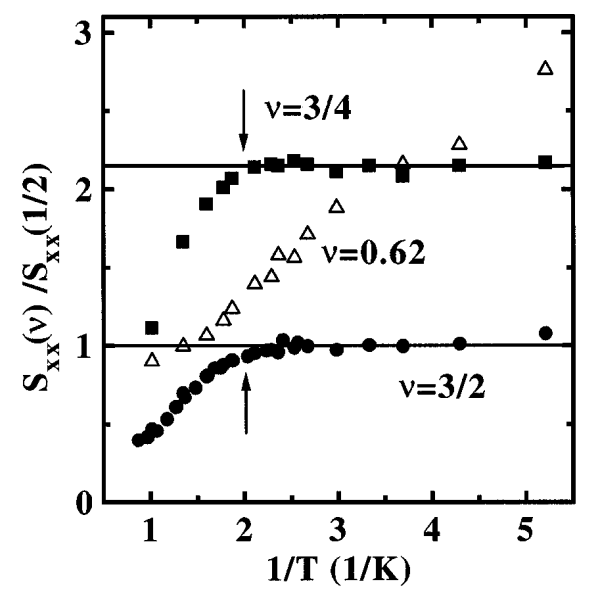

FIG. 2. The relative TEP $S_{x x}(\nu) / S_{x x}\left(\frac{1}{2}\right)$ at filling factors $\nu=$ $\frac{3}{2}$ and $\frac{3}{4}$ as a function of inverse temperature. Arrows indicate $T_{c}$, below which these ratios remain constant. Data for an arbitrary filling factor $(\nu=0.62$, a maximum in the TEP) are shown for comparison. observed in other samples before [7] and is not affected by the proximity of an insulating phase in our temperature range. We find $S_{x x}\left(\frac{1}{4}\right) / S_{x x}\left(\frac{1}{2}\right) \simeq 2.4$, which is close enough to $S_{x x}\left(\frac{3}{4}\right) / S_{x x}\left(\frac{1}{2}\right)$ to suggest that $S_{x x}\left(\frac{3}{4}\right) / S_{x x}\left(\frac{1}{4}\right)$ is also unity as for the $\frac{1}{2}$ states.

The absolute magnitude of the observed TEP varies strongly with temperature as can be seen in Fig. 3, which makes the constant relative magnitude of the TEP at evendenominator filling factors all the more remarkable. This figure also shows the strong similarity between $S_{x x}\left(\frac{1}{2}\right)$ and the zero-field TEP $S_{0}$ with the ratio $S_{x x}\left(\frac{1}{2}\right) / S_{0}$ being approximately constant. The power law behavior $S \propto T^{k}$ which is approached at low temperatures is very similar for $B=0(k=4.0 \pm 0.5)$ and $\nu=\frac{1}{2}(k=3.5 \pm 0.5)$, confirming that the TEP for both zero magnetic field and for even-denominator filling factors is caused by phonon drag. Furthermore, as seen in the inset of Fig. 3, the explicit density dependence of the absolute magnitude of the TEP at half filling at a fixed temperature is found to be $1 / n_{e}$.

A 2DEG at even-denominator filling factor can be regarded as a metallic state of CFs in zero effective magnetic field. This concept, together with the experimental results at even-denominator filling factors, strongly suggests that the coupling to the phonons is very similar for $\mathrm{CFs}$ and electrons. Therefore we use a model for the phonon drag TEP based on the solution of the Boltzmann equation for a $2 \mathrm{DEG}$ in zero magnetic field [9] to analyze our results. This calculation consists of two steps: the evaluation of (i) the thermally created drag current of quasiparticles and (ii) the electric field required to produce the compensating current. When $\nu=\frac{3}{2}$ and $\frac{3}{4}$ are

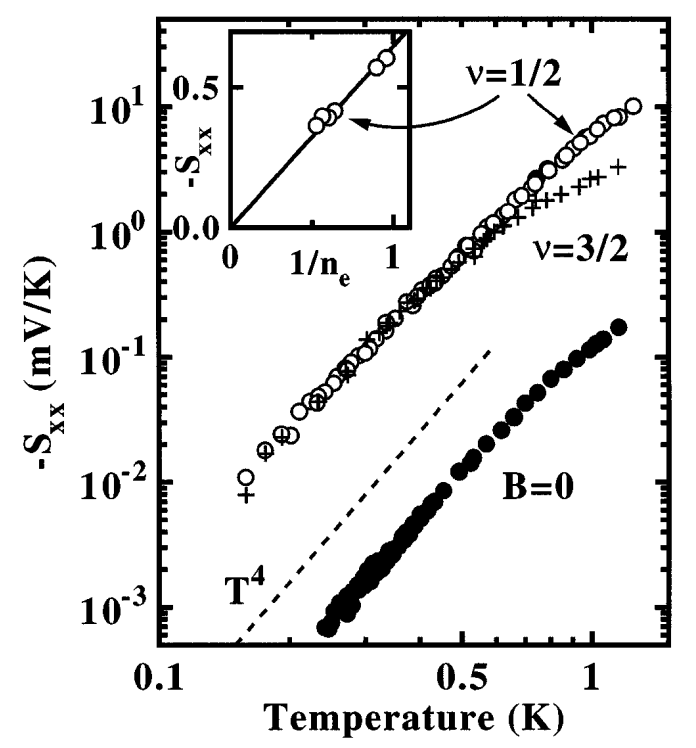

FIG. 3. The temperature dependence of the absolute value of the TEP for even-denominator filling factors and zero magnetic field. The dashed curve indicates a $T^{4}$ dependence. The inset shows $S_{x x}\left(\frac{1}{2}\right)$ at $T=440 \mathrm{mK}$ (in $\mathrm{mV} / \mathrm{K}$ ) versus the inverse density $1 / n_{e}$ (in units of $10^{-15} \mathrm{~m}^{2}$ ). 
considered, one must also account for the role of the filled Landau level to which the CF state is added. In this case, only a fraction $n_{Q} / n_{e}$ (where $n_{Q}$ is the CF density) of the total current $j_{\text {tot }}$ is carried by the quasiparticles [2]. In the derivation of Eq. (1) below, we have used the above relation between $j_{\text {tot }}$ and the CF current density $j_{Q}$, and have also made the assumption that the thermally created quasiparticle current must be compensated by a current of quasiparticles only. This gives the result that the total electron density $n_{e}$ appears in the prefactor; a behavior similar to the Hall resistivity which is also based on an effect of a compensation current.

For the range of temperatures and densities studied, typical phonon wave vectors $\left(q \sim 4 k T / \hbar v_{s}\right.$, where $v_{s}$ is the velocity of sound in GaAs) are small compared to the diameter of the Fermi circle for both electrons and CFs, and with this condition we obtain for the phonon drag TEP of CF states in zero effective magnetic field [9]

$$
S^{g}=-A V^{2} T^{4} \frac{m_{e}^{*}}{n_{e}} \iint \frac{m_{Q}^{*} v_{s}}{\hbar k_{F}^{Q}} f\left(\xi, \xi_{z}\right) d \xi d \xi_{z} .
$$

In this equation, $f\left(\xi, \xi_{z}\right)$ is a function containing the thermal distribution of the phonons and takes into account all possible scattering events with the quasiparticles consistent with energy and momentum conservation, with $\xi=q \hbar v_{s} / k T$ and $\xi_{z}=q_{z} \hbar v_{s} / k T$ being dimensionless phonon wave vectors in the plane of the 2DEG and perpendicular to it, respectively. The effective mass and Fermi wave vector of the CFs are denoted by $m_{Q}^{*}$ and $k_{F}^{Q}=\sqrt{4 \pi n_{Q}}$, and $m_{e}^{*}$ is the effective (band) mass of electrons in GaAs. The prefactor $A$ contains only fundamental constants and fixed parameters of the phonon system, and $V$ is an effective scattering potential for the CF-phonon coupling, which, in our case, may be taken to be constant [10]. The double integral is to be evaluated in the range $\sqrt{q^{2}+q_{z}^{2}} \leq 2 k_{F}$, and in its dimensionless form is almost independent of $T$ with our range of parameters.

In the CF model, the fundamental difference between $\nu=\frac{1}{2}$ and $\frac{3}{2}$ is that the quasiparticle density $n_{Q}$ differs by a factor of 3 for constant total electron density $n_{e}$ [2]. Since the integral contains only the ratio $m_{Q}^{*} / k_{F}^{Q}$, and both $m_{Q}^{*}$ and $k_{F}^{Q}$ are proportional to $\sqrt{n_{Q}}$ [2], $S^{g}$ becomes independent of $n_{Q}$ and we obtain $S^{g}\left(\frac{3}{2}\right)=S^{g}\left(\frac{1}{2}\right)$ and $S^{g}\left(\frac{3}{4}\right)=S^{g}\left(\frac{1}{4}\right)$ as observed experimentally. This result is independent of whether the $\nu=\frac{3}{2}$ state is totally polarized or only partially polarized, as recently observed [11] as long as $m_{Q}^{*}$ and $k_{F}^{Q}$ depend in the same way on the spin polarization, leaving the ratio $m_{Q}^{*} / k_{F}^{Q}$ unaltered. Furthermore, for the states with four flux quanta, it is plausible that the ratio $S_{x x}\left(\frac{3}{4}\right) / S_{x x}\left(\frac{1}{2}\right) \simeq 2.2$ arises from an increased effective mass $m_{Q}^{*}$ in Eq. (1) as observed in calculations [12] and other experiments [3] for $m^{*}\left(\frac{1}{4}\right)$. In any case, the TEP results clearly indicate that CF states with different numbers of flux quanta are indeed very similar.
Equation (1) not only describes the remarkable experimental fact that $S^{g}$ is independent of $n_{Q}$ for different even fractions with the same denominator, but also that $S^{g} \propto 1 / n_{e}$ at $\nu=\frac{1}{2}$ as seen in the inset of Fig. 3, whereas the experimental zero-field TEP $S_{0}$ is found to vary between $n_{e}^{-1.5}$ and $n_{e}^{-2}$. This observation can be explained by the fact that the electron mass $m_{e}^{*}$ does not depend on $n_{e}$, in contrast to $m_{Q}^{*}$ for CFs, so that Eq. (1) does indeed predict a stronger density dependence for electrons, $S_{0} \propto n_{e}^{-1} k_{F}^{-1} \propto n_{e}^{-1.5}$.

Focusing on the temperature dependence of electrons at zero magnetic field and CFs, they agree within experimental accuracy, $S_{0} \propto T^{4.0 \pm 0.5}$ and $S_{x x}\left(\frac{1}{2}\right) \propto T^{3.5 \pm 0.5}$. Equation (1) predicts a $T^{4}$ behavior for both particles in excellent agreement with this. In a recent paper, an evaluation of a related quantity, the phonon contribution $\mu_{\mathrm{ph}}$ to the electron (CF) mobility, has been inferred from $\rho_{x x}$ data, and a significant difference in $T$ dependence $\left(\mu_{\mathrm{ph}}^{\mathrm{CF}} \propto T^{-3}\right.$ and $\left.\mu_{\mathrm{ph}}^{e} \propto T^{-5}\right)$ has been found [13]. However, $S_{x x}^{g}$, which depends to first order on electron- (CF-) phonon scattering and, contrary to $\rho_{x x}$, is independent of impurity scattering, does not show this difference but, at most, an only marginally weaker $T$ dependence for CFs than for electrons.

Extending the comparison of the zero-field TEP with the CF states further, we find $S_{x x}\left(\frac{1}{2}\right) / S_{0}=50$ which would result in $m_{Q}^{*}\left(\frac{1}{2}\right) \approx 5 m_{0}$ (where $m_{0}$ is the free electron mass) if the magnitude of the change is attributed solely to mass enhancement. This is a very high value compared to those from Shubnikov-de Haas analyses which yield $m_{Q}^{*}\left(\frac{1}{2}\right) \approx m_{0}$ [3]. On the other hand, masses in the range $m_{Q}^{*}\left(\frac{1}{2}\right)=(2-6) m_{0}$ have also been reported from the mobility analysis mentioned above [13]. A possible explanation could be that the scattering potential $V$ in Eq. (1) differs not so much in $T$ dependence but in absolute value for $\mathrm{CFs}$ and electrons, but a more elaborate theory would be needed to confirm this statement.

Finally, we draw attention to the important feature that the TEP has a maximum at exactly half filling with an amplitude which increases with decreasing temperature and increasing density (see Fig. 4). This structure of the TEP is strikingly symmetric around $\nu=\frac{1}{2}$, showing that $S^{g}\left(B^{*}\right)=S^{g}\left(-B^{*}\right)$, where $B^{*}=B-B\left(\frac{1}{2}\right)$ is the effective magnetic field, a result completely consistent with the theoretical conjecture that $B\left(\frac{1}{2}\right)$ is an effectively zero magnetic field for CFs [2]. A similar symmetry is absent in the resistivity $\rho_{x x}$ where only a minimumlike structure on a rising background is observed (see top panel of Fig. 4). We attribute the lack of symmetry in the background of $\rho_{x x}$ to the fact that it is governed by the field dependence of $\tau_{i}$.

Using the result from Eq. (1) that $S \propto m_{Q}^{*}$, we can assign the symmetric peak in the TEP about $\nu=\frac{1}{2}$ to a property characteristic for the quasiparticle mass. In the CF theory, weak divergences of the form $m_{Q}^{*} \propto$ 


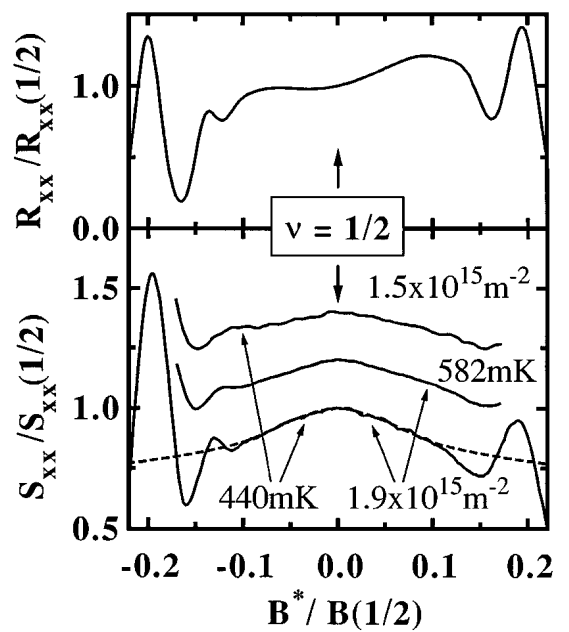

FIG. 4. The vicinity of $\nu=\frac{1}{2}$ : In the lower panel, $S_{x x}$, with the characteristic symmetric maximum at $\nu=\frac{1}{2}$, is shown for different temperatures and densities, respectively. The dashed line is a fit to the background of the lowest curve, assuming a logarithmically diverging mass at $\nu=\frac{1}{2}$. For comparison, the resistivity, which exhibits no similar symmetry around $\nu=\frac{1}{2}$, is shown in the upper panel.

$a^{\prime}+b^{\prime} /\left|B^{*}\right|^{0.5}$ or $m_{Q}^{*} \propto a+b \ln \left|B^{*}\right|$ for short range and Coulombic interactions, respectively, are expected as $\nu \rightarrow \frac{1}{2}[2,4]$. We have calculated the effect of a logarithmically diverging mass on the TEP using a simple distribution for the broadening of the effective field, $P(B)=$ $\left(1-\left|B-B_{0}\right| / \Delta B^{*}\right) / \Delta B^{*}$, and assuming that $B^{*}$ affects only $m_{Q}^{*}$, thus, $S\left(B_{0}\right) / S\left(\frac{1}{2}\right)=\int P(B) m^{*}(B) d B / m^{*}\left(\frac{1}{2}\right)$ for the normalized TEP. Using the fit parameters $b / a=0.16$ and $\Delta B^{*}=0.099$ [in units of $B\left(\frac{1}{2}\right)$ ], this function perfectly reproduces the smooth background in the vicinity of $\nu=\frac{1}{2}$ (see Fig. 4), demonstrating the consistency of the peak with a weakly diverging $m_{Q}^{*}$ as theoretically predicted. The value of $\Delta B^{*}$ is comparable to the width of the field region where no quantum oscillations are observed which coincides with the range of vanishing (or negative) gaps in activated transport measurements of $\rho_{x x}$ [3]. In a detailed analysis of these Shubnikov-de Haas experiments, a mass enhancement was also found as $\nu \rightarrow \frac{1}{2}$ [4], although these are not able to probe the region very close to $\nu=\frac{1}{2}$.

In conclusion, our experiments have shown that the TEPs of 2DEGs are identical for even-denominator filling factors with the same denominator and that the TEPs at different even denominators scale with a fixed constant. We have interpreted these findings by applying a zero-field theory for the phonon drag TEP to CFs. This approach provides an understanding not only of these results, but also of the temperature and density dependence of the TEP for both electrons and CFs, perhaps leaving only the high value of the ratio $S_{x x}\left(\frac{1}{2}\right) / S_{0}$ as an open question. Our results fully support the theoretical concept of CFs as fermions in an effective zero magnetic field. Finally, the symmetric peak in the TEP at $\nu=\frac{1}{2}$ is found to be in agreement with a weakly diverging effective mass of CFs at this filling factor.

[1] J. K. Jain, Adv. Phys. 41, 105 (1992).

[2] B. I. Halperin, P. A. Lee, and N. Read, Phys. Rev. B 47, 7312 (1993).

[3] R. R. Du, H. L. Stormer, D. C. Tsui, L. N. Pfeiffer, and K. W. West, Phys. Rev. Lett. 70, 2944 (1993); D. R. Leadley, R. J. Nicholas, C. T. Foxon, and J. J. Harris, Phys. Rev. Lett. 72, 1906 (1994).

[4] R. R. Du, H. L. Stormer, D. C. Tsui, L. N. Pfeiffer, and K. W. West, Phys. Rev. Lett. 73, 3274 (1994); P. T. Coleridge, Z. W. Wasilewski, P. Zawadzki, A. S. Sachrajda, and H. A. Carmona, Phys. Rev. B 52, R11 603 (1995).

[5] W. Kang, H.L. Stormer, L. N. Pfeiffer, W. Baldwin, and K. W. West, Phys. Rev. Lett. 71, 3850 (1993).

[6] R. L. Willett, R. R. Ruel, K. W. West, and L. N. Pfeiffer, Phys. Rev. Lett. 71, 3846 (1993).

[7] U. Zeitler, B. Tieke, S. A.J. Wiegers, J.C. Maan, R. Fletcher, V. I. Fal'ko, C. T. Foxon, and J. J. Harris, in High Magnetic Fields in the Physics of Semiconductors, edited by D. Heiman (World Scientific, Singapore, 1995), p. 38 .

[8] V. Bayot, E. Grivei, H.C. Manoharan, X. Ying, and M. Shayegan, Phys. Rev. B 52, R8621 (1995).

[9] D. G. Cantrell and P. N. Butcher, J. Phys. C 20, 1985 (1987); 20, 1993 (1987).

[10] It has been shown [S. K. Lyo, Phys. Rev. B 38, 6345 (1988)] that expressions such as Eq. (1) with a $T^{4}$ dependence are obtained for both unscreened deformation potential and screened piezoelectric scattering in the case of a 2DEG in zero magnetic field. In the case of deformation potential scattering, $V=E_{1}$, the deformation potential coefficient, while for piezoelectric scattering, $V$ is an effective potential.

[11] R. R. Du, A.S. Yeh, H. L. Stormer, D. C. Tsui, L. N. Pfeiffer, and K. W. West, Phys. Rev. Lett. 75, 3926 (1995).

[12] R. Morf and N. d'Ambrumenil, Phys. Rev. Lett. 74, 5116 (1995).

[13] W. Kang, Song He, H.L. Stormer, L. N. Pfeiffer, W. Baldwin, and K. W. West, Phys. Rev. Lett. 75, 4106 (1995). 